\title{
TOPOLOGICAL SPACES WITH THE FREESE-NATION PROPERTY II
}

\author{
JUDYTA BĄK AND ANDRZEJ KUCHARSKI
}

\begin{abstract}
The aim of this paper is to study the class of spaces with the FNS property and $\pi$ - FNS property. We shown that compact spaces with the FNS property for some base consisting of cozero-sets are openly generated spaces and spaces with the $\pi-$ FNS property are skeletally generated spaces.
\end{abstract}

\section{INTRODUCTION}

This paper is a continuation of the Freese-Nation property research introduced in the paper [1] for topological spaces. The Freese-Nation property was introduced by R. Freese and J.B. Nation [5]. In [1] it is shown that spaces with the FNS property satisfy ccc and any product of such spaces also satisfies ccc. All metrizable spaces have the FN property. L. Heindorf and L.B. Shapiro [9] showed that a family of all clopen sets of 0-dimensional compact space $X$ has the FNS property if and only if $X$ is openly generated. The concept of openly generated spaces was introduced by E.V. Shchepin in [20] and developed in [21] and [22]. The FNS property and some versions of it for compact spaces were studied in [9], [6], [7], [16], [17].

We say that a family $\mathcal{B}$ of open sets has FNS property if there exists a map s : $\mathcal{B} \rightarrow[\mathcal{B}]^{<\omega}$ such that if $U, V \in \mathcal{B}$ are disjoint then there are disjoint sets $W_{U}, W_{V} \in s(U) \cap s(V)$ such that $U \subseteq W_{U}, V \subset W_{V}$.

We say that a topological space $X$ has FNS property if there exists a base which has FNS property. We say that a space $X$ has $\pi-$ FNS property if there exists a $\pi$-base which has FNS property.

A topological space has the FN property if there exists a base $\mathcal{B}$ such that for every $V \in \mathcal{B}$ there are two finite sets $\mathrm{u}(V) \subseteq\{U \in \mathcal{B}: V \subseteq U\}$

2000 Mathematics Subject Classification. Primary: 54G20, 91A44; Secondary: $54 \mathrm{~F} 99$.

Key words and phrases. FNS property, inverse limit, openly generated space, skeletally generated space. 
and $\mathrm{l}(V) \subseteq\{U \in \mathcal{B}: U \subseteq V\}$ such that if $V \subseteq W$, then $\mathrm{u}(V) \cap \mathrm{l}(W) \neq$ $\emptyset$.

A regular space with countable weight has the FNS property and a regular space with countable $\pi$-weight has the $\pi$-FNS property. We estabilish in Section 2 that every compact Hausdorff space with the FNS property for some base consisting of cozero-sets is openly generated. We prove that Player I in open-open game has winning strategy in every space with the $\pi-$ FNS property. It follows from the result [11] that every compact Hausdorff space with the $\pi-$ FNS property is skeletally generated. In Section 3 we show that developable spaces have the FN property. We indicate examples of base without FNS property. Section 4 contains the proof that a space $Y$ which is co-absolute to a space $X$ with the $\pi-$ FNS property has the $\pi-$ FNS property.

\section{Openly Generated SPACED AND SKElEtally GENERARED SPACES}

We say that a compact space $X$ is openly generated if it is homeomorphic to $\lim _{\longleftarrow}\left\{X_{\sigma}, p_{\sigma}^{\sigma^{\prime}}, \Sigma\right\}$, where

(1) $X_{\sigma}$ is a compact metrizable space for $\sigma \in \Sigma$,

(2) $p_{\sigma}^{\sigma^{\prime}}: X_{\sigma^{\prime}} \rightarrow X_{\sigma}$ is an open surjection,

(3) $\Sigma$ is $\sigma$-complete, i.e. for any chain $\left\{\sigma_{n}: n \in \omega\right\} \subseteq \Sigma$ there exists $\sigma=\sup \left\{\sigma_{n}, n \in \omega\right\} \in \Sigma$,

(4) the inverse system is continuous, i.e. $X_{\sigma}=\lim _{\longleftarrow}\left\{X_{\sigma_{n}}, p_{\sigma_{n}}^{\sigma_{n+1}}, \omega\right\}$.

We say that a map $f: X \rightarrow Y$ is skeletal [15] if int $\operatorname{cl} f(U) \neq \emptyset$ for every nonempty open set $U \subseteq X$.

Similarly as openly generated space we define skeletally generated space, by replacing open surjections $p_{\sigma}^{\sigma^{\prime}}: X_{\sigma^{\prime}} \rightarrow X_{\sigma}$ with skeletal surjections. Skeletally generated spaces were introduced in [24].

Let $X$ and $Y$ be a topological spaces. We say that a function $f: X \rightarrow$ $Y$ is $d$-open [23] if $f(U) \subseteq \operatorname{int} \operatorname{cl} f(U)$ for every open set $U \subseteq X$.

Lemma 1 ([23]). Let $f: X \rightarrow Y$ and $f(U) \subseteq \operatorname{int} \mathrm{cl} f(U)$ for every set $U \in \mathcal{B}$, where $\mathcal{B}$ is a base of regular space $X$, a map $f$ is closed, then $f$ is open.

Lemma 2 ([12]). For any map $f: X \rightarrow Y$ the following conditions are equivalent:

(1) $f$ is d-open, 
(2) there exists a base $\mathcal{B}$ of a space $Y$ such that a family $\mathcal{P}=$ $\left\{f^{-1}(V): V \in \mathcal{B}\right\}$ satisfies the following condition: for every family $\mathcal{S} \subseteq \mathcal{P}$ and a point $x \notin \mathrm{cl} \cup \mathcal{S}$, there is an open neighborhood $W \in \mathcal{P}$ of $x$ such that $W \cap \bigcup \mathcal{S}=\emptyset$,

Let $\mathcal{P}$ be a family of open subsets of a space $X$. We introduce an equivalence relation $\sim_{\mathcal{P}}$ on a space $X$ in the following way

$$
x \sim_{\mathcal{P}} y \quad \Longleftrightarrow \quad(\forall V \in \mathcal{P} \quad x \in V \Leftrightarrow y \in V) .
$$

Let $X / \mathcal{P}$ be a set of all equivalence classes with the quotient topology and let $q_{\mathcal{P}}: X \rightarrow X / \mathcal{P}$ denote a quotient map (see [4]).

Following [10] (see also [2]) let us call a family $\mathcal{P}$ of open subsets of a spaces $X$ completely regular if for each $U \in \mathcal{P}$ there exist two sequences $\left\{V_{n}: n \in \omega\right\}$ and $\left\{U_{n}: n \in \omega\right\}$ in $\mathcal{P}$ such that

$$
U=\bigcup_{n \in \omega} U_{n} \text { and } U_{n} \subseteq X \backslash V_{n} \subseteq U \text { for each } n \in \omega \text {. }
$$

J. Kerstan has proved the following characterization of complete regularity.

Theorem ([10],(see also [2])). A $T_{1}$-space is completely regular if and only if it has a completely regular base.

We say that a family $\mathcal{P}$ of open subsets of a spaces $X$ weakly completely regular if for each $k \in \omega$ and any $U_{1}, \ldots, U_{k} \in \mathcal{P}$ there exist two sequences $\left\{\mathcal{A}_{n}: n \in \omega\right\},\left\{\mathcal{B}_{n}: n \in \omega\right\} \subseteq[\mathcal{P}]^{<\omega}$ such that

$$
\begin{aligned}
\text { (*) } U_{1} \cap \ldots \cap U_{k}=\bigcup\left\{\bigcup \mathcal{A}_{n}: n \in \omega\right\} & =\bigcup\left\{X \backslash \bigcup \mathcal{B}_{n}: n \in \omega\right\} \\
\text { and } \bigcup \mathcal{A}_{n} & \subseteq X \backslash \bigcup \mathcal{B}_{n} \text { for each } n \in \omega
\end{aligned}
$$

If a space $X$ has a completely regular family $\mathcal{P}$ then not so hard to see that $\mathcal{P}^{\prime}$ closed under the finite intersection and unions of elements of $\mathcal{P}$ is completely regular family. Then $\mathcal{P}^{\prime}$ is weakly completely regular family. Next theorem is similar to Theorem 2.16. [14] but we assume only that family $\mathcal{P}$ is a countable and weakly completely regular.

Theorem 1. If $\mathcal{P}$ is a countable and weakly completely regular family of $X$, then $X_{\mathcal{P}}$ is metrizable.

Proof. We will show that a map $q_{\mathcal{P}}: X \rightarrow X / \mathcal{P}$ is continuous. First we check that $\left\{q_{\mathcal{P}}[V]: V \in \mathcal{P}\right\}$ is a base of the space $X / \mathcal{P}$. To show that $\bigcup \mathcal{P}=X$, take any $x \in X$ and $W \in \mathcal{P}$. We are not losing generality assuming that $x \notin W$. Since $\mathcal{P}$ is weakly completely regular there is a 
sequence $\left\{\mathcal{B}_{n}: n \in \omega\right\} \subseteq[\mathcal{P}]^{<\omega}$ such that $W=\bigcup\left\{X \backslash \bigcup \mathcal{B}_{n}: n \in \omega\right\}$. Hence we get $x \in \bigcup \mathcal{B}_{0} \subseteq \bigcup \mathcal{P}$. This proves that $\bigcup\{q[V]: V \in \mathcal{P}\}=$ $X / \mathcal{P}$. Now we shall prove that

$$
q[V \cap W]=q[V] \cap q[W] \text { for all } U, W \in \mathcal{P} .
$$

Take any $V, W \in \mathcal{P}$. Clearly $q[V \cap W] \subseteq q[V] \cap q[W]$. Take an arbitrary $q[x] \in q[V] \cap q[W]$, then there is $y \in V$ such that $q[x]=q[y]$. By the definition of the relation $\sim_{\mathcal{P}}$ we get $x \in V$. The same reasoning shows that $x \in W$ so we proved that $q[x] \in q[V \cap W]$. Since $\mathcal{P}$ is the weakly completely regular family there is a sequence $\left\{\mathcal{A}_{n}: n \in \omega\right\} \subseteq[\mathcal{P}]^{<\omega}$ such that $V \cap W=\bigcup\left\{\bigcup \mathcal{A}_{n}: n \in \omega\right\}$. Therefore there exists $U \in$ $\bigcup\left\{\mathcal{A}_{n}: n \in \omega\right\}$ such that $q[x] \in q[U] \subseteq q[V \cap W]=q[V] \cap q[W]$. This proves that the family $\{q[V]: V \in \mathcal{P}\}$ is the base of the space $X / \mathcal{P}$.

The map $q$ is continuous, because $q^{-1}(q[V])=V$ for all $V \in \mathcal{P}$. To show that the space $X / \mathcal{P}$ is Hausdorff space, take $q[x] \neq q[y]$. There exists $W \in \mathcal{P}$ such that $x \in W$ and $y \notin W$. Since $\mathcal{P}$ is the weakly completely regular family there are sequences $\left\{\mathcal{A}_{n}: n \in \omega\right\},\left\{\mathcal{B}_{n}: n \in\right.$ $\omega\} \subseteq[\mathcal{P}]^{<\omega}$ which satisfy condition $(*)$ for the set $W$. There is $n \in \omega$ such that $x \in \bigcup \mathcal{A}_{n} \subseteq X \backslash \bigcup \mathcal{B}_{n} \subseteq W$ and $y \notin X \backslash \bigcup \mathcal{B}_{n}$. Therefore there are neighborhoods $U_{x} \in \mathcal{A}_{n} \subseteq \mathcal{P}$ of the point $x$ and $U_{y} \in \mathcal{B}_{n} \subseteq \mathcal{P}$ of the point $y$. Since $\bigcup \mathcal{A}_{n} \cap \bigcup \mathcal{B}_{n}=\emptyset$ we have $q\left[U_{x}\right] \cap q\left[U_{y}\right]=q\left[U_{x} \cap U_{y}\right]=\emptyset$ and $q[x] \in q\left[U_{x}\right], q[y] \in q\left[U_{y}\right]$.

We shall prove that $X / \mathcal{P}$ is regular. Take any closed set $F \subseteq X / \mathcal{P}$ and a point $q(x) \notin F$. Since $\left\{q_{\mathcal{P}}[V]: V \in \mathcal{P}\right\}$ is a base of the space $X / \mathcal{P}$ there is $V \in \mathcal{P}$ such that $q(x) \in q[V]$ and $q[V] \cap F=\emptyset$. By the property (*) of $\mathcal{P}$ there are sequences $\left\{\mathcal{A}_{n}: n \in \omega\right\},\left\{\mathcal{B}_{n}: n \in \omega\right\} \subseteq[\mathcal{P}]^{<\omega}$ which satisfy condition $(*)$ for the set $V$. So there is $n \in \omega$ such that $q(x) \in q[U] \in\left\{q[G]: G \in \mathcal{A}_{n}\right\}$ and $X / \mathcal{P} \backslash \bigcup\left\{q[G]: G \in \mathcal{B}_{n}\right\} \subseteq X / \mathcal{P} \backslash F$. Since $\cup \mathcal{A}_{n} \subseteq X \backslash \cup \mathcal{B}_{n}$ the space $X / \mathcal{P}$ is regular. By the Urysohn metrization theorem $X / \mathcal{P}$ is metrizable.

We get a similar characterization of complete regularity by the property $(*)$ obtained by J. Kerstan [10] ( see also [2]).

Corollary 1. A $T_{0}$-space is completely regular if and only if it has a weakly completely regular base.

Proof. Clearly the family of all cozero-set of a space is weakly comletely regular. Let $\mathcal{B}$ be a weakly completely regular base for a space $X$. To show that $X$ is completely regular take any $U \in \mathcal{B}$. One can assume that there is a countable subfamily $\mathcal{P} \subseteq \mathcal{B}$ such that $\mathcal{P}$ is weakly completely regular and $U \in \mathcal{P}$. By Theorem $1 X / \mathcal{P}$ is metrizable. 
Since $q^{-1}(q[V])=V$ for all $V \in \mathcal{P}$ and $q[U]$ is an open set in the metrizable space $X / \mathcal{P}, U$ is a cozero-set, this completes the proof.

Theorem 2. Every compact Hausdorff space with the FNS property for some base consisting of cozero-sets is openly generated.

Proof. Let $\mathcal{B}$ be a base consisting of cozero-sets of a space $X$ which has the FNS property. For every countable family $\mathcal{A} \subseteq \mathcal{B}$ there exists a countable weakly completely regular family $\mathcal{P}$ such that $\mathcal{A} \subseteq \mathcal{P} \subseteq \mathcal{B}$, and $s(V) \subseteq \mathcal{P}$ for all $V \in \mathcal{P}$. Indeed, if $U_{1}, \ldots, U_{k} \in \operatorname{coZ}(X)$, then $W=U_{1} \cap \ldots \cap U_{k} \in \operatorname{coZ}(X)$ and $W$ is $F_{\sigma}$ set. Therefore there exists a continuous function $f: X \rightarrow[0,1]$ such that

$$
W=f^{-1}((0,1])=\bigcup\left\{F_{n}: n \in \omega\right\},
$$

where $F_{n}=f^{-1}\left(\left[\frac{1}{n}, 1\right]\right) \subseteq f^{-1}\left(\left(\frac{1}{n+1}, 1\right]\right) \subseteq f^{-1}\left(\left[\frac{1}{n+1}, 1\right]\right)$.

Let $U_{n}=f^{-1}\left(\left(\frac{1}{n}, 1\right]\right)$, then $F_{n} \subseteq U_{n+1}$ for $n \in \omega$. Since the space $X$ is regular, for every $x \in F_{n}$ there exists $V_{x} \in \mathcal{B}$ such that $x \in V_{x} \subseteq \operatorname{cl} V_{x} \subseteq$ $U_{n+1}$. Set $F_{n}$ is compact for $n \in \omega$, hence $F_{n} \subseteq \bigcup\left\{V_{x_{i}}: i \leq k\right\} \subseteq$ $\bigcup\left\{\mathrm{cl} V_{x_{i}}: i \leq k\right\}=\operatorname{cl}\left(\bigcup\left\{V_{x_{i}}: i \leq k\right\}\right) \subseteq U_{n+1}$. Let $\mathcal{A}_{n}=\left\{V_{x_{i}}: i \leq k\right\}$. Similarly for $X \backslash U_{n+1} \subseteq X \backslash \operatorname{cl} \cup \mathcal{A}_{n}$ we get $\mathcal{B}_{n} \in[\mathcal{B}]^{<\omega}$ such that $X \backslash U_{n+1} \subseteq \bigcup \mathcal{B}_{n} \subseteq X \backslash \mathrm{cl} \cup \mathcal{A}_{n}$, then $\operatorname{cl} \bigcup \mathcal{A}_{n} \subseteq X \backslash \bigcup \mathcal{B}_{n} \subseteq U_{n+1}$. For every $n \in \omega$ we have $F_{n} \subseteq \bigcup \mathcal{A}_{n} \subseteq \operatorname{cl} \bigcup \mathcal{A}_{n} \subseteq X \backslash \bigcup \mathcal{B}_{n} \subseteq U_{n+1}$, hence $W=\bigcup\left\{F_{n}: n \in \omega\right\}=\bigcup\left\{\bigcup \mathcal{A}_{n}: n \in \omega\right\}=\bigcup\left\{X \backslash \bigcup \mathcal{B}_{n}: n \in \omega\right\}$.

By Theorem 1 the space $X / \mathcal{P}$ is metrizable. We shall show that $q: X \rightarrow X / \mathcal{P}$ is an open map. The map $q: X \rightarrow X / \mathcal{P}$ is closed map as a continuous map from a compact space to a Hausdorff space. According to the lemma 1 it is sufficient to show that a map $q$ is $\mathrm{d}$ open. By the lemma 2 a map $q: X \rightarrow X / \mathcal{P}$ is d-open if and only if a family $\mathcal{P}$ satisfies the condition 2 of this lemma. Take a family $\mathcal{S} \subseteq \mathcal{P}$ and $x \notin \operatorname{cl} \bigcup \mathcal{S}$. There exists $V \in \mathcal{B}$ such that $x \in V$ and $V \cap \bigcup \mathcal{S}=\emptyset$. Put $\mathcal{R}=\left\{V^{\prime \prime}: V \subseteq V^{\prime \prime}, V^{\prime \prime} \in s(V) \cap \mathcal{P}\right\}$. Since the base $\mathcal{B}$ has the FNS property there are sets $V^{\prime}, U^{\prime} \in s(V) \cap s(U) \subseteq \mathcal{P}$ such that $V \subseteq V^{\prime}$, $U \subseteq U^{\prime}$ and $V^{\prime} \cap U^{\prime}=\emptyset$ for every $U \in \mathcal{S}$. Therefore $\bigcap \mathcal{R} \cap \bigcup \mathcal{S}=\emptyset$. Since $\mathcal{P}$ is the weakly completely regular family there is a sequence $\left\{\bigcup \mathcal{A}_{n}: n \in \omega\right\} \subseteq[\mathcal{P}]^{<\omega}$ such that $\bigcap \mathcal{R}=\bigcup\left\{\bigcup \mathcal{A}_{n}: n \in \omega\right\}$. Since $x \in V \subseteq \bigcap \mathcal{R}$ there is $m \in \omega$ and $W \in \mathcal{A}_{m}$ such that $x \in W \subseteq \bigcap \mathcal{R}$. Hence $W \cap \bigcup \mathcal{S}=\emptyset$ this proves that the map $q$ is d-open. 
Put

$$
\begin{aligned}
\Sigma=\left\{\mathcal{P} \in[\mathcal{B}]^{<\omega}: \mathcal{P}\right. \text { is a weakly completely regular family } \\
\qquad \text { and } s(V) \subseteq \mathcal{P} \text { for all } V \in \mathcal{P}\} .
\end{aligned}
$$

The family $\Sigma$ ordered by inclusion is directed by the first part of the proof. If $\mathcal{P} \in \Sigma$ then a map $q: X \rightarrow X / \mathcal{P}$ is an open surjection onto the compact metrizable space $X / \mathcal{P}$. If $\mathcal{P} \subseteq \mathcal{R}$, where $\mathcal{P}, \mathcal{R} \in \Sigma$, then one can define a map $\pi_{\mathcal{P}}^{\mathcal{R}}: X_{\mathcal{R}} \rightarrow X_{\mathcal{P}}$ such that $\pi_{\mathcal{P}}^{\mathcal{R}} \circ q_{\mathcal{R}}=q_{\mathcal{P}}$. Clearly $\pi_{\mathcal{P}}^{\mathcal{R}}$ is open. If $\left\{\mathcal{P}_{n}: n \in \omega\right\}$ is an increasing chain in $\Sigma$ then the space $X_{\mathcal{P}}$, is homeomorphic to $\lim \left\{X_{\mathcal{P}_{n}}, \omega\right\}$, where $\mathcal{P}=\bigcup\left\{\mathcal{P}_{n}: n \in \omega\right\}$. Thus $\left\{X_{\mathcal{P}}, \pi_{\mathcal{P}}^{\mathcal{R}}, \Sigma\right\}$ is a $\sigma$-complete inverse system, all spaces $X_{\mathcal{P}}$ are compact and metrizable and all bonding maps $\pi_{\mathcal{P}}^{\mathcal{R}}$ are open. A map $h: X \rightarrow \lim \left\{X / \mathcal{P}, q_{\mathcal{P}_{1}}^{\mathcal{P}_{2}}, \Sigma\right\}$ given by the formula

$$
h(x)=\left\{[x]_{\mathcal{P}}\right\}_{\mathcal{P} \in \Sigma}
$$

is the homeomorphism.

Theorem 3. A family of all regular open sets in regular infinite space does not have the FNS property.

Proof. Suppose that a regular infinite space $X$ has a map s: $\mathrm{RO}(X) \rightarrow$ $[\mathrm{RO}(X)]^{<\omega}$ witnessing the FNS property for $\mathrm{RO}(X)$. Consider the Stone space $\operatorname{Ult}(\mathrm{RO}(X))$ of the Boole'a algebra $\mathrm{RO}(X)$ with a topology generated by a base

$$
\mathcal{B}=\{\bar{U}: U \in \operatorname{RO}(X)\}
$$

where

$$
\bar{U}=\{\mathcal{F} \in \operatorname{Ult}(\operatorname{RO}(X)): U \in \mathcal{F}\} .
$$

Let $\overline{\mathrm{s}}: \mathcal{B} \rightarrow[\mathcal{B}]^{<\omega}$ be given by the formula

$$
\overline{\mathrm{s}}(\bar{U})=\{\bar{W}: W \in \mathrm{s}(U)\} .
$$

Since $\bar{U} \cap \bar{V}=\overline{U \cap V}$ for all $U, V \in \operatorname{RO}(X)$, the family $\mathcal{B}$ has the FNS property.

$\operatorname{Ult}(\mathrm{RO}(X))$ is the compact Hausdorff space with the FNS property. By Theorem $2 \operatorname{Ult}(\mathrm{RO}(X))$ is openly generated and $\operatorname{Ult}(\mathrm{RO}(X))=$ $\lim _{\longleftarrow}\left\{X_{\sigma}, \mathrm{p}_{\rho}^{\sigma}, \Sigma\right\}$, where $\mathcal{S}=\left\{X_{\sigma}, \mathrm{p}_{\sigma}^{\rho}, \Sigma\right\}$ is a $\sigma$-complete inverse system,

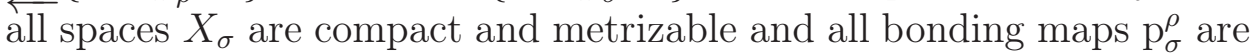
open. Since the space $\operatorname{Ult}(\mathrm{RO}(X))$ is extremally disconnected and each $\mathrm{p}_{\sigma}$ is an open map, every $X_{\sigma}$ is extremally disconnected. The space $X_{\sigma}$ is extremally disconnected and compact metric, therefore it has to be finite. 
Since $X$ is the infinite regular space, $|\operatorname{RO}(X)|>\omega$. For each $n \in \omega$ there exists $\sigma_{n} \in \Sigma$ such that $\mathrm{w}\left(X_{\sigma_{n}}\right)>n$ and $\left\{\sigma_{n}: n \in \omega\right\}$ is a chain. Since $\mathcal{S}$ is the $\sigma$-complete inverse system $\mathrm{w}\left(X_{\sigma}\right) \geq \omega$ where $\sigma=\sup \left\{\sigma_{n}: n \in \omega\right\}$, a contradiction.

Corollary 2. A topology of regular infinite space does not have the FNS property.

Proof. Assume that the topology of regular infinite space $X$ has the FNS property. From the Proposition 2.2 [1] it follows that the family of all regular open sets $\mathrm{RO}(X)$ has the FNS property, a contardiction with Proposition 3.

From the above proposition follows that $\beta \mathbb{N}$ has the $\pi-$ FNS property and does not have the FNS property.

Example 1. Suppose that there exists a base $\mathcal{B}$ of $\beta \mathbb{N}$ with the FNS property. Then $\mathcal{B}^{\prime}=\{\mathrm{cl} U: U \in \mathcal{B}\}$ is a base consisting of clopen sets. It's easy to see that $\mathcal{B}^{\prime}$ has the FNS property. By Theorem 2 there exists a $\sigma$-complete inverse system $\mathcal{S}=\left\{X_{\sigma}, \mathrm{p}_{\sigma}^{\rho}, \Sigma\right\}$ such that all spaces $X_{\sigma}$ are compact and metrizable and all maps $\mathrm{p}_{\sigma}^{\rho}, \mathrm{p}_{\sigma}$ are open and $\lim \mathcal{S}=\beta \mathbb{N}$, a contradiction because $\beta \mathbb{N}$ is extremally disconnected (compare the proof of Theorem 3). Since $\beta \mathbb{N}$ has a countable $\pi$ - base $\mathcal{B}$ closed under the complement it easy to define operator s : $\mathcal{B} \rightarrow[\mathcal{B}]^{<\omega}$ which is witness on the FNS property (compare [1, Proposition 5]).

Corollary 3. If $X$ is a regular infinite space, then the base $\mathrm{RO}(X)$ does not have the FNS property.

Now we shall prove that if $X$ is a regular infinite space, then the bases $\mathrm{RO}(X)$ and the topology of $X$ do not have the FN property.

Consider a cardinal number $\kappa>\omega$ with the discrete topology. It easy to see that a base $\{\{\alpha\}: \alpha<\kappa\}$ has the FN property. One can check that $\mathrm{u}(\{\alpha\})=\{\{\alpha\}\}=\mathrm{l}(\{\alpha\})$ for all $\alpha<\kappa$ have the desired properties. Next theorem gvies a negative answer to the question: Does the base $\mathrm{RO}(X)$ ( topology) have the FN property, whenever there exists some base with FN property?

Theorem 4. A family of all regular open sets in regular infinite space does not have the FN property.

Proof. Let $X$ be a regular infinite space. Suppose that operators $\mathrm{u}, \mathrm{l}$ are witnesses on the FN property for a family of all regular open sets $\mathrm{RO}(X)$. Since the space $X$ is regular and infinite there is an infinite 
maximal family $\mathcal{R} \subseteq \mathrm{RO}(X)$ of pairwise disjoint set. We may define by a straight forward recursion a sequence $\left\{V_{n}: n \in \omega\right\} \subseteq \operatorname{RO}(X)$ and a sequence of finite sets $\left\{\mathcal{P}_{n} \in[\mathcal{R}]^{<\omega}: n \in \omega\right\}$ such that

(1) $V_{n} \subsetneq V_{n+1}$ and $\mathcal{P}_{n} \subsetneq \mathcal{P}_{n+1}$ for all $n \in \omega$,

(2) $V_{n} \subseteq \bigcup\left(\mathcal{R} \backslash \mathcal{P}_{n}\right)$ for all $n \in \omega$,

(3) $V_{n} \cap W=\emptyset$ or $W \subseteq V_{n}$ for all $W \in \mathcal{R}$ and $n \in \omega$,

(4) $\left\{W \in \mathcal{R}: W \subseteq V_{n}\right\}$ is finite for all $n \in \omega$,

(5) If $V_{n} \subseteq W \subseteq \bigcup\left(\mathcal{R} \backslash \mathcal{P}_{n}\right)$, then $V_{n} \in \mathrm{l}(W)$, for each $W \in \mathcal{B}$ and $n \in \omega$.

Suppose that we have just defined $\mathcal{P}_{i} \in[\mathcal{R}]^{<\omega}$ and $V_{i} \in \operatorname{RO}(X)$ with the property $(1)-(5)$ for $i \leq n$. For each $W \in \mathrm{u}\left(V_{n}\right) \backslash\left\{V_{n}\right\}$ there is $U \in \mathcal{R}$ such that $W \cap U \neq \emptyset$ and $U \cap V_{n}=\emptyset$. Therefore there is a finite family $\mathcal{P}_{n+1} \subseteq \mathcal{R}$ such that $\mathcal{P}_{n} \subseteq \mathcal{P}_{n+1}$ and $\bigcup \mathcal{P}_{n+1} \cap W \neq \emptyset$, whenever $W \in \mathrm{u}\left(V_{n}\right) \backslash\left\{V_{n}\right\}$. Finaly we get $\left\{V_{n}: n \in \omega\right\} \subseteq \mathrm{RO}(X)$ and $\left\{\mathcal{P}_{n} \in[\mathcal{R}]^{<\omega}: n \in \omega\right\}$ which satisfy properties $(1)-(5)$. Let $\mathcal{P}=\bigcup\left\{\mathcal{P}_{n}: n \in \omega\right\}$ and $U=\operatorname{int} \operatorname{cl} \bigcup\left\{V_{n}: n \in \omega\right\}$. For every $n \in \omega$ we get

$$
V_{n} \subseteq \bigcup\left\{V_{n}: n \in \omega\right\} \subseteq U \subseteq \bigcup(\mathcal{R} \backslash \mathcal{P}) \subseteq \bigcup\left(\mathcal{R} \backslash \mathcal{P}_{n}\right)
$$

Hence $V_{n} \in \mathrm{l}(U)$ for every $n \in \omega$ by property (5), a contradiction.

Corollary 4. A topology of regular infinite space does not have the FN property.

Proof. Assume that the topology of regular infinite space $X$ has the FN property. Suppose that operators u, l are witnesses on the FN property for a family of all open sets. Put

$\mathrm{u}_{\text {reg }}(U)=\{\operatorname{int} \mathrm{cl} W: W \in \mathrm{u}(U)\}$ and $\mathrm{l}_{\text {reg }}(U)=\{\operatorname{int} \mathrm{cl} W: W \in \mathrm{l}(U)\}$,

for every $U \in \operatorname{RO}(X)$. The operators $\mathrm{u}_{r e g}, \mathrm{l}_{\text {reg }}$ are witnesses on the FN property for a family of all regular open sets, a contradition with Theorem 4.

In the paper [1] we have proved that if there exists a base $\mathcal{B}$ closed under the finite intersections with the FNS property, then one can enlarge the base $\mathcal{B}$ to a base $\mathcal{B}^{\prime}$ with FNS that contains both families $\mathcal{B}$ and $\operatorname{Clop}(X)$. Now we can strengthen the mentioned result.

Theorem 5. If a 0-dimensional compact space $X$ has the FNS property for some base consisting of cozero-sets, then $\operatorname{Clop}(X)$ has the FNS property. 
Proof. By Theorem $2 X$ is openly genereted space, i.e. $\mathcal{S}=\left\{X_{\mathcal{P}}, \pi_{\mathcal{P}}^{\mathcal{R}}, \Sigma\right\}$ is a $\sigma$-complete inverse system, all spaces $X_{\mathcal{P}}$ are compact and metrizable and all bonding maps $\pi_{\mathcal{P}}^{\mathcal{R}}$ are open and $X=\lim \mathcal{S}$. We may assume without loss of generality that $X_{\mathcal{P}}$ are 0 -dimensional. Applying Heindorf and Shapiro's result [9, Theorem 2.2.3] ( see also [1, Theorem 21]) we get an operator which witnesses the FNS property for the family $\operatorname{Clop}(X)$.

The open-open game was introduced by P. Daniels, K. Kunen and H. Zhou [3] for an arbitrary topological space $X$. Two players take countably many turns. A round consists of player I choosing a nonempty set $U$ and player II choosing a nonempty $V$ with $V \subseteq U$. Player I wins if the union of II's open sets is dense in $X$, otherwise player II wins. Denote this game by $G(X)$. Consider the following game $G_{\omega}(X)$ on a topological space $X$. At the $n$-th round Player I chooses a finite family $\mathcal{C}_{n}$ of open non-empty open sets. Then Player II chooses a finite family $\mathcal{D}_{n}$ of non-empty open subsets of $X$ such that for each $U \in \mathcal{C}_{n}$ there exists $V \in \mathcal{D}_{n}$ with $V \subseteq U$. Player I wins if the union of II's open sets is dense in $X$, otherwise player II wins. It's well known that the game $G(X)$ is equvalent to the game $G_{\omega}(X)$ (see [3]). We say that Player I has a winning strategy in the game $G_{\omega}(X)$ whenever there exists a function

$$
\left(\mathcal{D}_{0}, \mathcal{D}_{1}, \ldots, \mathcal{D}_{n}\right) \mapsto \sigma\left(\mathcal{D}_{0}, \mathcal{D}_{1}, \ldots, \mathcal{D}_{n}\right),
$$

where all families $\mathcal{D}_{n}$ and $\sigma\left(\mathcal{D}_{0}, \mathcal{D}_{1}, \ldots, \mathcal{D}_{n}\right)$ are finite and consists of non-empty open sets such that for each game

$$
\sigma(\varnothing), \mathcal{D}_{0}, \sigma\left(\mathcal{D}_{0}\right), \mathcal{D}_{1}, \sigma\left(\mathcal{D}_{0}, \mathcal{D}_{1}\right), \mathcal{D}_{2}, \ldots, \mathcal{D}_{n}, \sigma\left(\mathcal{D}_{0}, \ldots, \mathcal{D}_{n}\right), \mathcal{D}_{n+1}, \ldots
$$

the union $\bigcup_{n \geq 0} \cup \mathcal{D}_{n}$ is dense in $X$. Assume that $\mathcal{B}$ is an $\pi-$ base, then I Player has a winning strategy in the game $G(X)$ if and only if there is a winning strategy defined on $\pi$ - base $\mathcal{B}$ ( see [3]).

Theorem 6. In every compact Hausdorff space with the $\pi-$ FNS property I Player has a winning strategy in open-open game.

Proof. Assume that $X$ is a compact Hausdorff space there are $\pi$ - base $\mathcal{B}$ and $\mathrm{s}: \mathcal{B} \rightarrow[\mathcal{B}]^{<\omega}$ which witnessing the $\pi-$ FNS property. We shall define a winning strategy $\sigma$ for I player in the game $G_{\omega}(X)$. Take any $U_{0} \in \mathcal{B}$ and put $\mathcal{C}_{0}=\sigma(\emptyset)=\left\{U_{0}\right\}$. Assume that we have just defined

$$
\sigma(\emptyset), \mathcal{D}_{0}, \ldots, \sigma\left(\mathcal{D}_{0}, \ldots, \mathcal{D}_{n-1}\right), \mathcal{D}_{n}
$$

where all families $\mathcal{D}_{j}$ and $\sigma\left(\mathcal{D}_{0}, \mathcal{D}_{1}, \ldots, \mathcal{D}_{j}\right)$ are finite and consists of non-empty open sets such for each $U \in \sigma\left(\mathcal{D}_{0}, \mathcal{D}_{1}, \ldots, \mathcal{D}_{j}\right)$ there exists

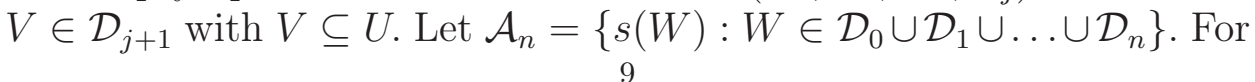


each finite family $\mathcal{R} \subseteq \mathcal{A}_{n}$ with the non-empty intersection we choose $V_{\mathcal{R}} \in \mathcal{B}$ such that $V_{\mathcal{R}} \subseteq \bigcap \mathcal{R}$. Put

$$
\sigma\left(\mathcal{D}_{0}, \ldots, \mathcal{D}_{n}\right)=\left\{V_{\mathcal{R}} \in \mathcal{B}: \mathcal{R} \in\left[\mathcal{A}_{n}\right]^{<\omega} \text { and } \bigcap \mathcal{R} \neq \emptyset\right\}
$$

Suppose that $\bigcup_{n \in \omega} \cup \mathcal{D}_{n}$ is not dense in $X$. There is a set $V \in \mathcal{B}$ such that $V \cap \bigcup_{n \in \omega} \cup \mathcal{D}_{n}=\emptyset$. For each $U \in \bigcup_{n \in \omega} \cup \mathcal{D}_{n}$ there is $W \in s(V) \cap s(U)$ such that $W \cap U=\emptyset$ and $V \subseteq W$. There is $n \in \omega$ such that

$$
\mathcal{R}=\left\{W \in s(V): \exists\left(U \in \bigcup_{n \in \omega} \bigcup_{\mathcal{D}_{n}}\right) W \cap U=\emptyset \text { and } V \subseteq W\right\} \subseteq \mathcal{A}_{n} .
$$

Hence there exists $V \in \mathcal{D}_{n+1}$ such that $V \subseteq \bigcap \mathcal{R}$, a contradiction with $\bigcap \mathcal{R} \cap \bigcup_{n \in \omega} \cup \mathcal{D}_{n}=\emptyset$.

Similar theorem to the Theorem 2 is true for spaces with $\pi-$ FNS property and skeletaly generated spaces.

Theorem 7. Every compact Hausdorff space with the $\pi-\mathrm{FNS}$ property is skeletally generated.

Proof. Assume that $X$ is compact Hausdorff space with the $\pi-$ FNS property. By Theorem 6 I Player has a winning strategy. Using [11, Theorem 12] we get $X$ is skeletally generated.

\section{Developable spaces have the FN property}

Let $\mathcal{U}$ be a cover of a space $X$ and $x \in X$. Recall [4] that a set $\operatorname{St}(x, \mathcal{U})=\bigcup\{U \in \mathcal{U}: x \in U\}$ is called a star of the point $x$ with respect to the cover $\mathcal{U}$. A sequence $\left\{\mathcal{W}_{n}\right\}_{n \in \omega}$ of covers of a space $X$ is called a development whenever for every point $x \in X$ and an open set $U \subseteq X$ such that $x \in U$ there exists $i \in \omega$ such that $\operatorname{St}\left(x, \mathcal{W}_{i}\right) \subseteq U$. A space which has a development is called developable.

A family $\mathcal{U}=\left\{U_{t}: t \in T\right\}$ of sets is called a point-finite if for every $x \in X$ the set $\left\{t \in T: x \in U_{t}\right\}$ is finite. We say that a cover $\mathcal{U}=\left\{U_{t}: t \in T\right\}$ is a refinement of a cover $\mathcal{V}=\left\{V_{s}: s \in S\right\}$ if for every $t \in T$ there exists $s \in S$ such that $U_{t} \subseteq V_{s}$.

Proposition 8. A topological space having a development consisting of point-finite covers has FN property.

Proof. Let $\left\{\mathcal{U}_{n}\right\}_{n \in \omega}$ be a a development consisting of point-finite covers of $X$. Without loss of generality it can be assumed that there exists 
a development $\left\{\mathcal{W}_{n}\right\}_{n \in \omega}$ consisting of point-finite covers such that the cover $\mathcal{W}_{n+1}$ is a refinement of the cover $\mathcal{W}_{n}$.

For a family $\mathcal{A}$ of sets we shall denote by $\mathcal{A}^{M}$ subfamily of $\mathcal{A}$ consisting of all maximal elements, i.e. of set $A \in \mathcal{A}$ such that if $A \subseteq A^{\prime} \in \mathcal{A}$ then $A=A^{\prime}$. Since each $\mathcal{W}_{n}$ is point-finite cover $\mathcal{W}_{n}^{M}$ is well defined and it is the point-finite cover. Two distinct elements in $\mathcal{W}_{n}^{M}$ are incomparable by the inclusion. The sequence $\left\{\mathcal{W}_{n}^{M}\right\}_{n \in \omega}$ is the development. Indeed, take a point $x \in X$ and a neighborhood $V$ of $x$. Since $\left\{\mathcal{W}_{n}\right\}_{n \in \omega}$ is the development there is $n \in \omega$ such that $\operatorname{St}\left(x, \mathcal{W}_{n}\right) \subseteq V$. If $x \in W \in \mathcal{W}_{n}^{M} \subseteq \mathcal{W}_{n}$ then $W \subseteq U$. Therefore $\operatorname{St}\left(x, \mathcal{W}_{n}^{M}\right) \subseteq V$. The family $\mathcal{B}=\bigcup\left\{\mathcal{W}_{n}^{M}: n \in \omega\right\}$ is a base for the space $X$. For each $U \in \mathcal{B}$ put

$\mathrm{l}(U)=\{U\}$ and $\mathrm{u}(U)=\left\{W \in \mathcal{W}_{i}^{M}: U \subseteq W, i \leq \min \left\{n: U \in \mathcal{W}_{n}^{M}\right\}\right\}$.

If $U \subseteq V$, then $U \in \mathcal{W}_{k}^{M}$ and $V \in \mathcal{W}_{n}^{M}$ for $k<n$ or $n<k$. If $k<n$, then for $V$ there is $W \in \mathcal{W}_{k}^{M}$ such that $U \subseteq V \subseteq W$. Since elements of $\mathcal{W}_{k}^{M}$ are incomparable by the inclusion, we have $U=V$. If $n<k$, then $V \in l(V) \cap u(U)$.

Since every metrizable space has a development consisting of pointfinite covers [4, Theorem 5.4.8] we get the following

Corollary 5 ([1]). Metric spaces satisfy FN property.

\section{FNS PROPERTY AND ABSOLUTES}

We will need a notion of a small image and some facts connected with it.

Let $f$ be a map from a space $X$ to a space $Y$. A small image of a set $U \subseteq X$ under a map $f$ is called a set $f^{\#}(U)=\left\{y \in Y: f^{-1}(y) \subseteq U\right\}$.

Lemma 3. Let $f$ be a map from $X$ to $Y$, then small image of a set $U \subseteq X$ under the map $f$ is given by $f^{\#}(U)=Y \backslash f(X \backslash U)$.

Proof. We will show that $Y \backslash f(X \backslash U)=\left\{y \in Y: f^{-1}(y) \subseteq U\right\}$ for every set $U \subseteq X$.

Let $y \in Y \backslash f(X \backslash U)$, then $y \in Y$ and for every $x \in X$ such that $y=$ $f(x)$ we have $x \notin X \backslash U$, hence $y \in Y$ and $f^{-1}(y) \subseteq U$.

Let $y \in Y$ and $f^{-1}(y) \subseteq U$, then $f^{-1}(y) \cap(X \backslash U)=\emptyset$. Hence $f\left(f^{-1}(y) \cap(X \backslash U)\right)=\{y\} \cap f(X \backslash U)=\emptyset$ and $\{y\} \subseteq Y \backslash f(X \backslash U)$. 
Lemma 4. Let $f: Z \rightarrow X$ be an irreducible map and $U, V \subseteq Z$ be open sets, then $U \cap V=\emptyset$ if and only if $f^{\#}(U) \cap f^{\#}(V)=\emptyset$.

Proof. Let $U, V \subseteq Z$ and $U \cap V=\emptyset$. Suppose that $f^{\#}(U) \cap f^{\#}(V) \neq \emptyset$, then there exists $y \in Y$ such that $f^{-1}(y) \subseteq U \cap V$, this is a contradiction.

Let $f^{\#}(U) \cap f^{\#}(V)=\emptyset$. Suppose that $U \cap V \neq \emptyset$, then we get $\emptyset \neq X \backslash f(Z \backslash(U \cap V))=f^{\#}(U \cap V) \subseteq f^{\#}(U) \cap f^{\#}(V)$ and this contradiction ends the proof.

Lemma 5. Let $f$ be a map from $Z$ onto $X$. For every set $U \subseteq Z$ we have $f^{\#}(U) \subseteq f(U)$.

Proof. Let us take any set $U \subseteq Z$ and $y \in f^{\#}(U)$, then $f^{-1}(y) \subseteq U$. Hence there exists $x \in f^{-1}(y) \subseteq U$ and $y=f(x) \in f(U)$.

We say that a continuous, closed map $f$ from $X$ onto $Y$ is irreducible if $Y$ is not the image of any proper closed subset of $X$. The absolute of a space $X$ is the space which is mapped and irreducibly onto $X$, and is such that any irreducible inverse image of the space $X$ is homeomorphic to it. We say that topological spaces are coabsolute if their absolutes are homeomorphic.

Lemma 6. If $\mathcal{B}_{X}$ is a $\pi$-base for $X$ and $f: Z \rightarrow X$ is an irreducible and closed map, then $\left\{f^{-1}(B): B \in \mathcal{B}_{X}\right\}$ is a $\pi$-base for $Z$.

Proof. We will show that for every open set $\emptyset \neq U \subseteq Z$ there exists a set $B \in \mathcal{B}_{X}$ such that $f^{-1}(B) \subseteq U$. Let us take any nonempty, open set $U \subseteq Z$, then $Z \backslash U \neq Z$ and it is closed. Hence $X \backslash f(Z \backslash U) \neq \emptyset$ and it is open, then there exists an open set $B \in \mathcal{B}_{X}$ such that $B \subseteq$ $X \backslash f(Z \backslash U)$. We get $\emptyset=B \cap f(Z \backslash U)=f\left(f^{-1}(B) \cap(Z \backslash U)\right)$. Hence $f^{-1}(B) \cap(Z \backslash U)=\emptyset$, and $f^{-1}(B) \subseteq U$.

Theorem 9. Every space $Y$ which is co-absolute to a space $X$ with the $\pi$ - FNS property has the $\pi-$ FNS property.

Proof. Let a space $Z$ be an absolute of the space $X$ and $g: Z \rightarrow X$, $f: Z \rightarrow Y$ be irreducible, onto maps. Let $\mathcal{B}_{X}$ be a $\pi$-base for $X$ which has FNS property and s witnesses this property.

We will show that the family $\left\{f^{\#} g^{-1}(V): V \in \mathcal{B}_{X}\right\}$ is a $\pi$-base for $Y$. Let $U$ be an open set in $Y$. By Lemma 6 there exists a set $V \in \mathcal{B}_{X}$ such that $g^{-1}(V) \subseteq f^{-1}(U)$. Hence by Lemma $5 f^{\#} g^{-1}(V) \subseteq$ $f^{\#} f^{-1}(U) \subseteq f f^{-1}(U) \subseteq U$. 
We will show that the family $\left\{f^{\#} g^{-1}(V): V \in \mathcal{B}_{X}\right\}$ has FNS property. We define a map s $\mathrm{s}_{Z}:\left\{f^{\#} g^{-1}(V): V \in \mathcal{B}_{X}\right\} \rightarrow\left[\left\{f^{\#} g^{-1}(V): V \in\right.\right.$ $\left.\left.\mathcal{B}_{X}\right\}\right]^{<\omega}$ by the formula

$$
\mathrm{s}_{Z}\left(f^{\#} g^{-1}(U)\right)=\left\{f^{\#} g^{-1}(W): W \in \mathrm{s}(U)\right\} .
$$

Let $f^{\#} g^{-1}(V) \cap f^{\#} g^{-1}(U)=\emptyset$ for some $V, U \in \mathcal{B}_{X}$. By Lemma 4 it has to be $V \cap U=\emptyset$, then there exist disjoint sets $V^{\prime}, U^{\prime} \in \mathrm{s}(U) \cap$ $s(V)$. We have $f^{\#} g^{-1}\left(V^{\prime}\right), f^{\#} g^{-1}\left(U^{\prime}\right) \in s\left(f^{\#} g^{-1}(V)\right) \cap \mathrm{s}\left(f^{\#} g^{-1}(U)\right)$, $f^{\#} g^{-1}(V) \subseteq f^{\#} g^{-1}\left(V^{\prime}\right)$ and $f^{\#} g^{-1}(U) \subseteq f^{\#} g^{-1}\left(U^{\prime}\right)$. By Lemma 4 we have $f^{\#} g^{-1}\left(V^{\prime}\right) \cap f^{\#} g^{-1}\left(U^{\prime}\right)=\emptyset$. This ends the proof.

Dugundji spaces were introduced in [18]. Skeletally Dugundji spaces are skeletal analogue of Dugundji spaces, see [13]. We say that a compact space $X$ is Dugundji (skeletally Dugundji) whenever $X$ can be represented as the limit space of a continuous inverse system $S=$ $\left\{X_{\alpha}, p_{\alpha}^{\beta}, \alpha<\beta<w(X)\right\}$ such that $X_{0}$ is a compact metrizable with open bonding (skeletal) maps and each map $p_{\alpha}^{\alpha+1}$ has a metrizable kernel, see [8]. L. Shapiro [19] proved that every skeletally Dugundji spaces are co-absolute to a 0-dimensional Dugundji spaces. By Theorem 2.2.3 [9] ( see also Theorem 21 [1]) and Theorem 9 every 0-dimensional skeletally Dugundji space has the $\pi-$ FNS property.

Question 1. Do openly generated spaces have the FNS property?

Question 2. Do skeletally generated spaces have the $\pi-\mathrm{FNS}$ property?

\section{REFERENCES}

[1] J. Bąk and A. Kucharski, Topological spaces with the Freese-Nation property, accepted in Annales Mathematicae Silesianae. 1, 2, 1, 2, 2, 5, 4

[2] H. Brandenburg and A. Mysior, Short proof of an internal characterization of complete regularity, Canad. Math. Bull. 27 (1984), no. 4, 461-462. 2, 2

[3] P. Daniels, K. Kunen and H. Zhou, On the open-open game, Fund. Math. 145 (1994), no. 3, $205-220.2$

[4] R. Engelking, General topology, Heldermann Verlag, Berlin (1989). 2, 3, 3

[5] R. Freese and J. B. Nation, Projective lattices, Pacific Journal of Mathematics, 75 (1975), 93-106. 1

[6] S. Fuchino, S. Koppelberg and S. Shelah, Partial orderings with the weak Freese-Nation property, Ann. Pure Appl. Logic 80 (1996), 35-54. 1

[7] S. Fuchino and L. Soukup, More set-theory around the weak Freese-Nation property, Fund. Math. 154 (1997), 159-176. 1

[8] R. Haydon, On a problem of Pelczynski: Milutin spaces, Dugundji spaces and AE $(0-\operatorname{dim})$, Studia Math. 52 (1974), 23-31. 4

[9] L. Heindorf, L. B. Shapiro, Nearly Projective Boolean Algebras, Lecture Notes in Math., vol. 1596, (1994). 1, 2, 4 
[10] J. Kerstan, Eine Charakterisierung der vollständig regulären Räume, Math. Nachr. 17 (1958), 27-46. 2, 2

[11] A. Kucharski and Sz. Plewik, Inverse systems and I-favorable spaces, Topology Appl. 156 (2008), no. 1, 110-116. 1, 2

[12] A. Kucharski, Sz. Plewik and V. Valov, Game theoretic approach to skeletally Dugundji and Dugundji spaces, Topology Appl. 201 (2016), 206-216. 2

[13] A. Kucharski, Sz. Plewik and V. Valov, Skeletally Dugundji spaces, Central Europ. J. Math. 11 (2013), 1949-1959. 4

[14] A. Kucharski and S. Turek, A generalization of $\varkappa$-metrizable spaces, preprint available at arXiv:1612.08838 2

[15] J. Mioduszewski and L. Rudolf, $H$-closed and extremally disconnected Hausdorff spaces, Dissertationes Math. 66 (1969). 2

[16] D. Milovich, On the strong Freese-Nation property, Order 34 (2017), no. 1, 91-111. 1

[17] D. Milovich, The $(\lambda, \kappa)$-Freese-Nation property for Boolean algebras and compacta, Order 29, 361-379 (2012). 1

[18] A. Pełczyński, Linear extensions, linear averagings, and their applications to linear topological classification of spaces of continuous functions, Dissert. Math. 58 (1968), 1- 89. 4

[19] L. Shapiro, On spaces co-absolute with dyadic compacta, Dokl. Acad. Nauk SSSR 293 (1987), no. 6, 1077-1081 (in Russian). 4

[20] E.V. Shchepin, Topology of limit spaces with uncountable inverse spectra, Uspekhi Mat. Nauk 31 (1976), no. 5(191), 191-226. 1

[21] E. V. Shchepin, On $\kappa$-metrizable spaces, (Russian) Izv. Akad. Nauk SSSR Ser. Mat. 43 (1979), no. 2, 442-478. 1

[22] E. V. Shchepin, Functors and uncountable powers of compacta, Uspekhi Mat. Nauk 36 (1981), no. 3(219), 3-62. 1

[23] M. Tkachenko, Some results on inverse spectra II, Comment. Math. Univ. Carolin. 22 (1981) 819-841. 2, 1

[24] V. Valov, External characterization of I-favorable spaces, Mathematica Balkanica 25 (2011), no. 1-2, 61-78.

2

Judyta Bąk, Institute of Mathematics, Jan Kochanowski UniverSity, Świętokrzyska 15, 25-406 Kielce and Institute of Mathematics, University of Silesia, Bankowa 14, 40-007 Katowice, Poland

E-mail address: jubak@us.edu.pl

Andrzej Kucharski, Institute of Mathematics, University of Silesia in Katowice, Bankowa 14, 40-007 Katowice, Poland

E-mail address: andrzej.kucharski@us.edu.pl 